\title{
Study on Information Integration of Condition Monitoring and Fault Diagnosis System in Manufacturing
}

\author{
Liu Dan', Xu Guanghua ${ }^{2}$, Liang Lin ${ }^{2}$, and Luo Ailing ${ }^{2}$ \\ 1 Department of Instrument Science and Technology, School of \\ Mechanical Engineering, Xi'an Jiaotong University, Xi'an 710049, China \\ jxegg@stu.xjtu.edu.cn \\ 2 Department of Instrument Science and Technology, School of \\ Mechanical Engineering, Xi'an Jiaotong University, Xi'an 710049, China
}

\begin{abstract}
Aimed at the problems of equipment condition information share and integration in the enterprise monitoring and diagnosis systems, the XML-based model of equipment condition information was presented by analysing the structure of equipment condition information. Afterwards, with the help of the model, the idea of intranet-based software "bus" for machine monitoring and fault diagnosis system was proposed, which is a criterion that specifies the data presentation, management and communication protocol. According to the difference of work mode, the information provider is divided into pusher and puller, the information applicant passively or actively receives the information correspondingly. Then, the technique has been applied to the information integration between the portable condition monitoring system and the online condition monitoring system, Results show that it have enough flexibility and expansibility, and can be applied to large-scale information integration of equipment condition information.
\end{abstract}

\section{Introduction}

Machinery information integration has the potential to rationalize and improve business processes in oil refinery, metallurgy, petrifaction, and other industries. With the development of technologies, many condition monitoring and fault diagnosis systems of various corporations have been applied in industries, which leads to disordered phenomena in enterprises, such as various information sources, different 
information formats and representations. Simultaneously, with the development of remote monitoring and diagnosis technology [1-6], enterprise presses for a method to support machinery information integration.

A key challenge in any information integration task is to resolve the heterogeneity among the semantic terminologies used in the various information sources to be integrated [7]. Another key challenge is to resolve the information communication in the various condition monitoring and diagnosis systems.

Aimed at challenges above, firstly, this paper analyzes the structure of equipment condition information and presents an XML-based model of equipment condition information to resolve the problem of information represent, then, puts forward an information integration framework to realize the information communication. Section 4 describes a prototype implementation of the framework. Finally section 5 concludes the paper.

\section{Machinery Condition Information Representation}

Traditional representation of machinery condition information uses text file or database. Its main drawback is that representation has strong dependency on application system. Meanwhile, it is difficult to solve the integration of heterogeneous information and heterogeneous environment. For example, it is difficult to represent pictures using text file. For all, MIMOSA (Machinery Information Management Open Systems Alliance) puts forward a CRIS criterion and defines a machinery information database criterion, but almost all companies don't completely follow the database criterion in application, like Bentley Company in USA and Strongwish Company in China.

The Extensible Markup Language (XML) [8], a World Wide Web Consortium (W3C) [9] Standard, has been widely accepted for storing and exchanging structured and semi-structured documents. Many XML sublanguages have been developed to define constrained data in XML format for special application areas, often by means of a Document Type Declaration (DTD) or XML Schema [10], definition. For example, Mathematical Markup Language (MathML) [11], is defined for electronic interchange of mathematical symbols, equations and formulae or Voice Extensible Markup Language (VoiceXML) [12], is developed for voice markup and telephony call control to enable access to the Web using spoken interaction. Such markup languages are becoming increasingly popular because $\mathrm{XML}$ is simple, easy to understand, extensible, searchable, open standard, interoperable and there is a wide range of tool support for creation, manipulation and transformation of $X M L$ documents automatically [13]. In this section we discuss machinery condition information representation using XML based on generic machinery condition information model. 


\section{Machinery Condition Information Model}

In the first step, the machinery condition information representation of individual mechanical component is modeled using UML. The classes in the UML model are encoded the machinery condition information nodes.

The second step is to identify the functionally equivalent constructs in different machinery component and generalize them at a higher level of abstraction. Fig. 1 presents a part of generic model for machinery information.

The UML diagram is a graphical representation of the model. In third step, for storage and model interchange, the UML models are encoded in XML DTD definition. This results in one DTD for each of the representations in various machinery condition monitoring and fault diagnosis system. The produced DTD and the XML representations of the machinery condition information are instances of them that will be validated against the models.

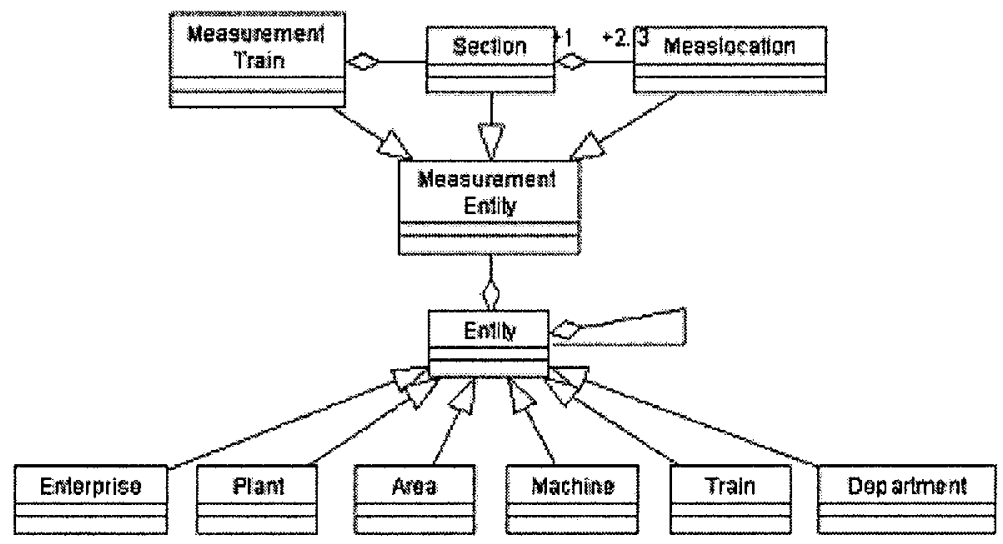

Fig. 1. Generic Machinery Information Model

\section{Information Integration System Framework}

In Fig. 2 we present the multilayer framework for information integration system of condition monitoring and fault diagnosis system. We also demonstrate the usage of the framework for building the information integration system. The framework is composed of three parts: Adapters for various systems, Software bus framework, Location Service. Adapter for various systems components are the representation transformers, Software bus framework is the transfer and Location Service component provides location information of various adapter components. 
The aim of the framework is to let users focus on specifying what they want, rather than thinking about how to obtain the answers. As a result, it frees them from the tedious tasks of finding relevant data sources, interacting with each source in isolation using a particular interface, and combining data from multiple sources.

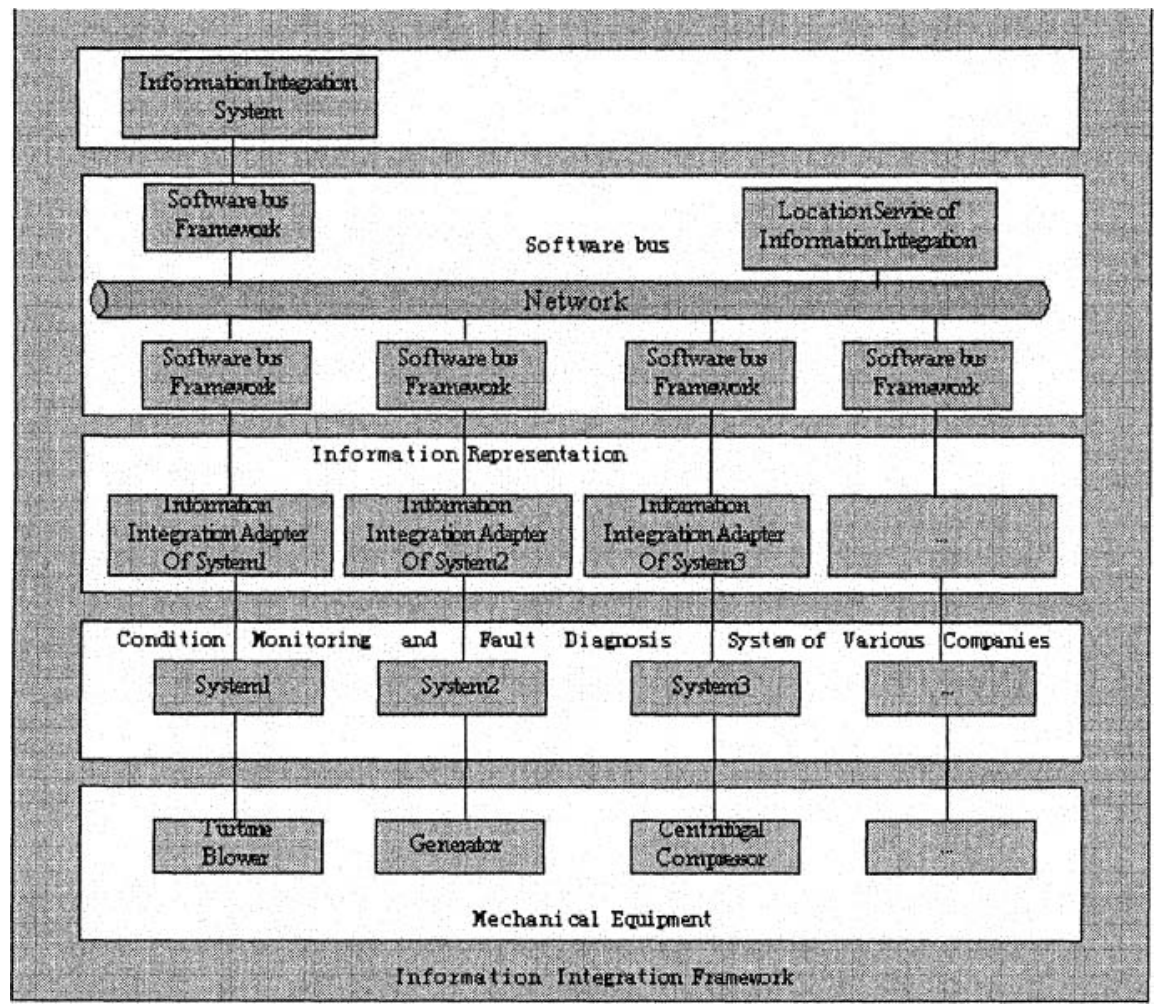

Fig. 2. System structure of Information Integration System

\subsection{Adapters}

A set of adapter of various systems is required to convert the representations from various systems to the machinery condition information model of XML abstraction. It takes charge of encoding the data in history database (history data) or memory database (real-time data) using XML and transferring the XML document stream to software bus framework. So, it must be developed correspondingly according to different condition monitoring and fault diagnosis systems. 


\subsection{Software Bus Framework}

Software bus framework is required to transmit XML document stream through physical network. It is consists of application development interface and a daemon service. Fig. 3 shows the software bus framework architecture. Daemon service provides the communication function through Intranet. Application development interface called by the adapter component is required to build the associations between the adapter component and daemon service and control behaviors of daemon service.

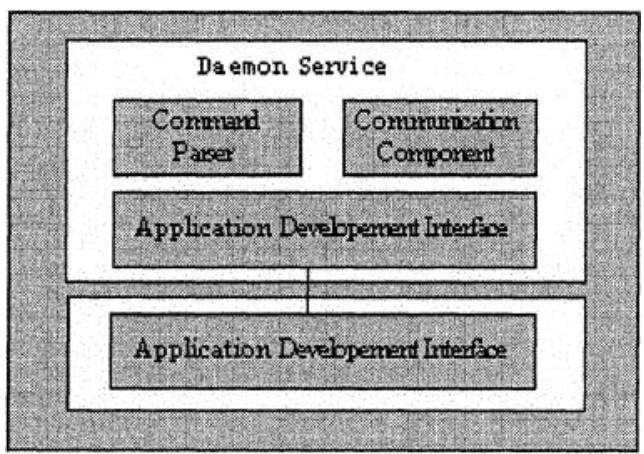

Fig. 3. Components of Software Bus Framework

Daemon Service is divided into two parts: Command Parser (CP) and Communication Component (CC). $\mathrm{CP}$ is required to interpret and execute the command from other daemon services. $\mathrm{CC}$ takes charge of sending and receiving command or data through physical network.

Daemon service as an information provider of information integration system works in three modes: push mode, pull mode and mix mode.

1) Push mode: is used to transfer real-time data of online system. When it works in this mode, information integration system must register to it. So, it could notify information integration system when new data are coming.

2) Pull mode: is used to transfer history data of condition monitoring and fault diagnosis system. When it works in this mode, information integration system submit query requirement to daemon service using address obtained through location service component.

3) Mix mode: provides functions both push mode and pull mode.

According to the difference of its work mode, the information applicant passively or actively receives the information correspondingly. 


\subsection{Location Service}

Location service component is required to maintain and update the address of adapters. When an adapter starts, it must notify location service component and update itself address using software bus framework component. Meanwhile, location service component is required to query status of the adapter with some time interval to refresh the address list.

\section{A Prototype Implementation}

We have developed a prototype based on structure above. Our prototype is an information integration system that integrates information of both a portable measurement system (It installed on notebook has a portable measurement hardware.) and an online monitoring system. Figure 4 shows the structure of system. Firstly, adapter component for each of monitoring system is developed correspondingly. Then an information integration system is developed using information integration system framework. Figure 5 shows the main user interface of information integration system. In control panel of the main user interface, a machine tree is displayed using tree view control, which shows structures of both portable system and online system. The right panel shows a wave after FFT of a measure point.

The prototype was developed using the $\mathrm{C \#}$ programming language and all the experiments were run in an Intel $42.40 \mathrm{G} \mathrm{MHz}$ with $512 \mathrm{MB}$ of RAM and running Microsoft Windows 2000 Operating System. It's about $400 \mathrm{~ms}$ to query a history data with 1024 dot using the system. The time required to query data is quite reasonable and depends on the size of history data. 


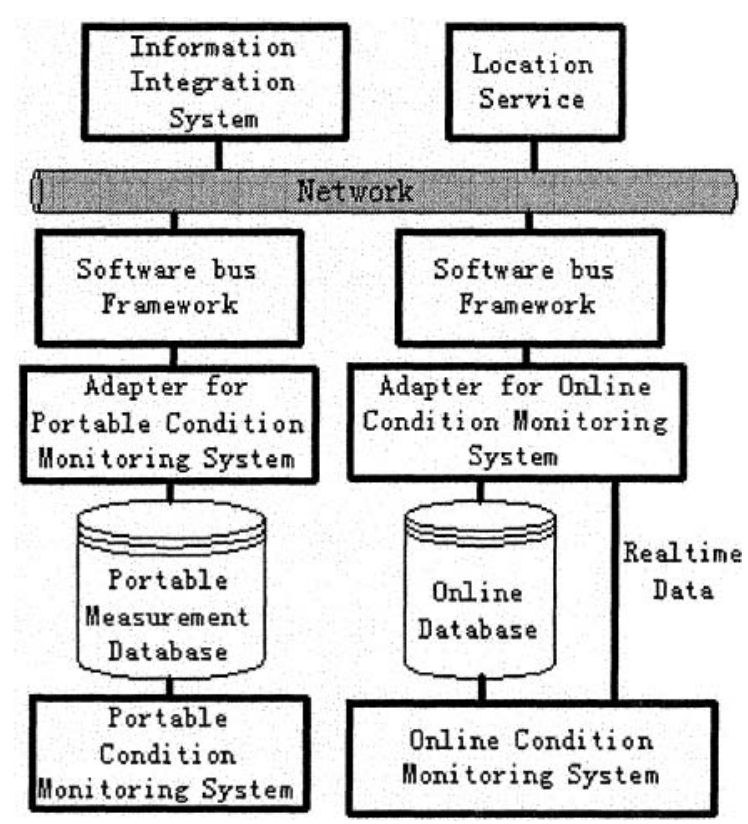

Fig. 4. System structure of the Implementation

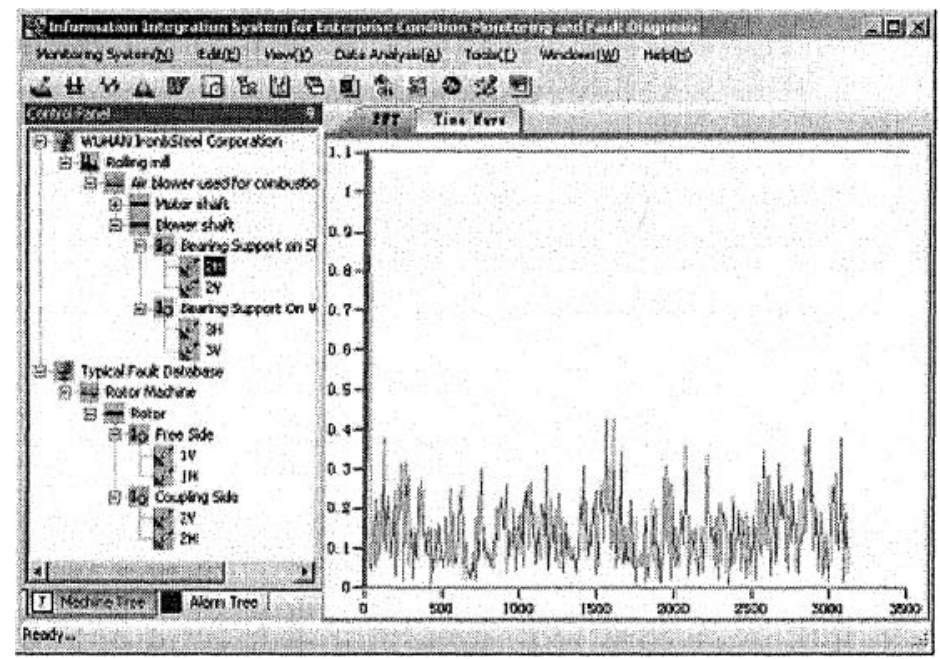

Fig. 5. Main UI of Information Integration System 


\section{Conclusions}

In this paper we presented a framework for information integration of condition monitoring and fault diagnosis system. The framework is based on abstraction of machinery condition information using XML. With the help of the model, the idea of Intranet-based software "bus" for machine monitoring and fault diagnosis system was proposed, which is a criterion that specifies the data presentation, management and communication protocol. According to the difference of work mode, the information provider is divided into pusher and puller, the information applicant passively or actively receives the information correspondingly.

The obtained results of the prototype system show that the framework and with reasonable performance. As a conclusion, this paper provides the fundamental mechanism to build information integration system of machinery condition that will perform machinery condition analysis independently of the specifically application system. Information fusion of machinery condition based on the mechanism is the next target.

\section{References}

1. R. Itschner, C. Pommerell, and M. Rutishauser, GLASS Remote Monitoring of Embedded Systems in Power Engineering, IEEE Internet Computing 2(5), 46-52 (1998).

2. H. S. Li, L. S. He, T. L. Shi, and et al, Research of B/S-based Remote Diagnostics Expert System, Journal of WUHAN University of Technology 21(4), 39-41 (1999).

3. Y. Xiaohong and Q. Liangsheng, Design of the Internet-based Machinery Diagnosis Center, CHINA Mechanical Engineering 5, 874-876 (2002).

4. H. Lingsong, W. Junfeng, and Y. Shuzi, Internet Based Remote Coooperative Diagnostics for Machine Faults, CHINA Mechanical Engineering 3, 336-338 (1999).

5. P.W. Tse and L.S. He, Web and Virtual Instrument Based Machine Remote Sensing, Monitoring and Fault Diagnostic System, Proceedings of the ASME Design Engineering Technical Conference, Pittsburgh, ASME, 2001, pp. 2919-2926.

6. T. Zhang and H. Zuo, Study of Several Key Technical Problems for Civil Aero engine Remote Diagnosis, Journal of Aerospace Power China 18(1), 32-37 (2003).

7. R. Al-Ekram and K. Kontogiannis, An XML-based Framework for Language Neutral Program Representation and Generic Analysis, IEEE Proceeding of the Ninth European Conference on Software Maintenance and Reengineering, 2005.

8. XML.ORG (Nov 7, 2005); www.xml.org.

9. World Wide Web Consortium (Nov 7, 2005); www.w3c.org.

10. XML Schema (Nov 7, 2005); www.w3.org/XML/Schema.

11. MathML (Nov 7, 2005); www.w3.org/Math.

12. VoiceXML (Nov 7, 2005); www.w3.org/TR/voicexml20.

13. C.E. Kuziemsky and F. Lau, Ontology-based Information Integration in Health Care: A Focus on Palliative Care, IEEE Proceedings of the Eleventh Annual International Workshop on Software Technology and Engineering Practice, 2004. 